\title{
Gas-filled Umbilical Hernia (Pneumo-umbilicus). A CT Sign of Hypertensive Pneumoperitoneum
}

Several signs of pneumoperitoneum, related to the diaphragmatic domes, right upper quadrant, lesser sac, and lower abdomen, have been recognised by using first plain abdominal radiographs and later Computed Tomography (CT).

These findings are especially evident when an abundant, eventually tension, valvular pneumoperitoneum is present (Addison N et al, Br J Surg 1976; 63 : 877). In this case the gas under pressure distends the peritoneal spaces, ligaments and folds, elevates the diaphragm, compresses the viscera, and eventually dissects through all possible pathways, including the retroperitoneum or a patent inguinal canal (Bray JF, Br J Radiol 1982; 55: 867, Cho KC et al, Radiology
1997; 202: 651, Grassi R et al, Br J Radiol 1996; 69: 774). We add another occurrence; an encapsulated gas collection within the umbilical area demonstrated by helical $\mathrm{CT}$ in a subject with tension pneumoperitoneum. To the best of our knowledge this sign, which we named "pneumo-umbilicus", has not been reported previously.

\section{Case report}

A 75-year-old woman was admitted to the emergency room with sudden diffuse abdominal pain, abdominal distension, dyspnoea, and hypotension.

The patient did not have a pre-existing umbilical hernia but a large tension 
outpouching, that had suddenly developed in relationship with the abdominal distenston, was evident within the umbilical area at physical inspection.

An unenhanced helical CT examination was promptly performed. The scout view (Fig. 1) showed a large amount of intraperitoneal gas and a mid-abdominal well-demarcated round lucency. The CT study (Fig. 2 a, b) confirmed the abundant free abdominal gas with posterior displacement of the bowel loops. At level of the umbilicus a gas-filled oval sac was evident, with a thin and well-defined border. The umbilical ring showed a subtle interruption at the ligamentum teres insertion, probably representing the site through which the intraperitoneal gas under pressure had reached the abdominal wall.

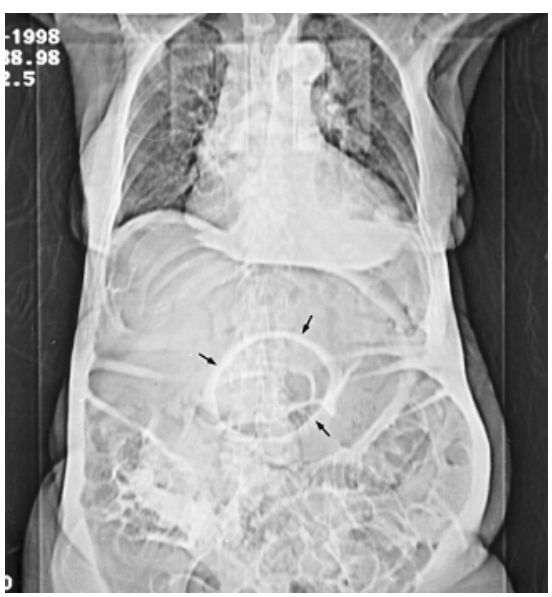

Fig. 1 Scout-view. A large amount of free intra-abdominal gas distends the peritoneal spaces. A round and well-defined lucency is evident within the umbilical area (arrows).

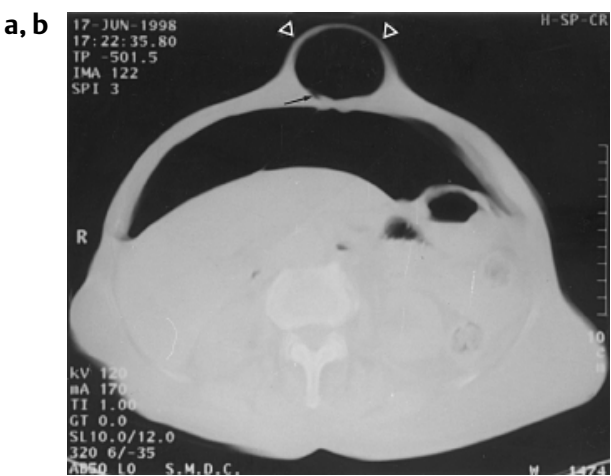

Fig. 2 (a,b) Unenhanced helical CT of the abdomen (two non-contiguous images at lung window setting). Abundant pneumoperitoneum with dorsal compression of the abdominal viscera. An oval-shaped gas collection is
A redundant transverse colon was also noted with a marked and diffuse wall thickening and with obliterated surrounding fat planes. Both ovaries appeared enlarged and inhomogeneous. Bilateral pleural effusion and mild peritoneal effusion were finally evident. At surgery a grossly perforated colonic metastasis from ovarian adenocarcinoma was found and a resection with direct anastomosis was carried out. The umbilical outpouching was composed of parietal peritoneum and was easily reduced. The patient was unaware of the ovarian cancer but retrospectively weight loss and change in bowel habits (mucous diarrhea) were found in the history.

\section{Discussion}

The umbilicus is a scar representing the site of attachment of the umbilical cord in the foetus. The umbilical fibrotic ring is supported by the round ligament (that crosses posteriorly to reach the inferior border) and by the urachus and medial umbilical ligaments (also inserted at the lower border). The ring is posteriorly reinforced by the Richet umbilical fascia, that may leave its upper part uncovered.

If the umbilical ring falls to close properly or if the upper portion of the umbilicus is particularly weak, congenital or acquired abnormalities may develop. Umbilical hemia is the most common occurrence in adulthood and is usually acquired, developing secondarily to obesity, multiple pregnancies, chronic bowel distension, intra-abdominal masses or increased intra-abdominal pressure (ascites).

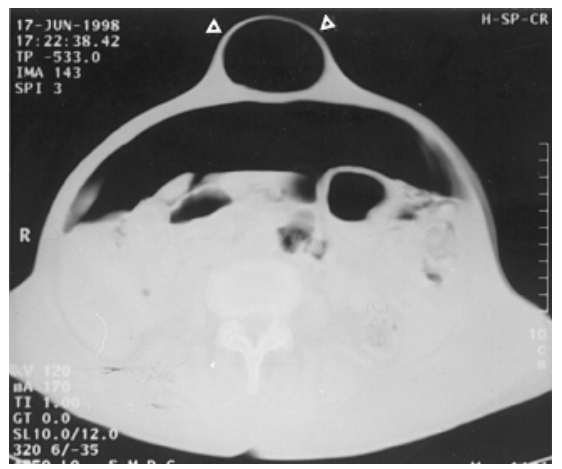

clearly appreciable within the abdominal wall, creating an evident outpouching of the anterior surface (white arrows). A small interruption (black arrow in a) is visible at the level of the ligamentum teres insertion.
Umbilical hernias are described on supine plain film as dense oval-shaped masses with a partially ill defined contour that is typical of abdominal wall abnormalities (the so called "incomplete border sign" absent in intra-abdominal abnormalities) (Mendelson E, Radiol Clin North Am 1964; 2 :161). Our pattern, as recognisable on the scout-view, is in contrast with this finding since the oval lucency had a well-defined border all around.

CT may optimally depict abdominal wall hemias and their content. In our case the umbilical gas-filled hernia and its significance was clearly demonstrated by helical CT scanning.

In this patient with large pneumoperitoneum emergency laparotomy would have been performed anyway. Nevertheless, the clinical-radiological presentation of a tension pneumoperitoneum is complex and requires a prompt but accurate assessment.

In a minority of patients with gastrointestinal perforation a valvular mechanism may develop with air entering the peritoneal cavity and causing a dramatic increase in the intraabdominal pressure. Usually, this may cause an elevation of the diaphragm and of the lower pulmonary lobes recognisable on plain abdominal films. In addition, a central compression of the gut may occur. However, we describe for the first time that in rare cases this elevation of the intraabdominal pressure may be associated with a gas-filled umbilical hernia. Thus, the finding of a gas-filled umbilical hernia may be regarded as a farther CT sign of hypertensive pneumoperitoneum.

Hypertensive pneumoperitoneum is a life-threatening condition giving rise to an abdominal compartment syndrome that should be promptly communicated to surgeons because of necessity of emergency management (Pickhardt PJ et al, AM 1999; 173 - 575). In some cases it may be necessary to insert a trocar or a large bore needle to decompress preoperatively the abdomen, similarly to the emergency relief of a tension pneumothorax (Yao DC et al, AJR 1997; 168 : 1389).

O. Catalano, M. Esposito, A. Siani, Pozzuoli, Italy 